\title{
Time-of-day variation in the diagnostic quality of screening colonoscopies: a registry-based study
}

\author{
File Jaho, Rasmus Kroijer, Magnus Ploug
}

Hospital South West Jutland, Esbjerg, Denmark

\begin{abstract}
Background The diagnostic quality of screening colonoscopies has been found to differ between morning and afternoon. Specifically, the adenoma detection rate (ADR) is higher in the morning. Our aim was to assess if time-of-day dependent differences in colonoscopy quality exist in a Danish screening setting. Following national screening guidelines, an individual will be exempt from screening invitations for 8 years if the colonoscopy is without pathology. Therefore, it is of utmost importance to identify factors systematically affecting the detection of lesions.
\end{abstract}

Methods This was a single-center study of screening colonoscopies performed between 2014 and 2018. Records were retrieved from the Danish Colorectal Cancer Screening Database and coupled with local data. The ADR and the cecal intubation rate were compared between morning (8-12 a.m.) and afternoon (12-4 p.m.) colonoscopies. Multivariate logistic regression analysis was performed.

Results A total of 3659 screening colonoscopies were included. The ADR was $51 \%$ in the morning and $58 \%$ in the afternoon. Multivariate analysis found this statistically significant, with the "afternoon vs. morning" odds ratio for adenoma detection being 1.4 (95\% confidence interval 1.17-1.68; $\mathrm{P}<0.001$ ). The cecal intubation rate was $95.6 \%$ in the morning and $94.7 \%$, a non-significant difference.

Conclusions The ADR of screening colonoscopies was higher in the afternoon. Our study highlights the need for local/regional evaluation of factors affecting colonoscopy quality to address such issues. A clean colonoscopy exempts the patient from subsequent screening invitations for 8 years. Therefore, any observed systematic differences in quality must be addressed and eliminated.

Keywords Colorectal cancer, screening, endoscopy, colonoscopy, adenoma detection rate

Ann Gastroenterol 2021; 34 (1): 1-5

\section{Introduction}

Colorectal cancer (CRC) screening was introduced in Denmark in 2014 in order to reduce CRC incidence and mortality [1]. Citizens aged 50-74 years are offered a single fecal immunochemical test (FIT) biannually, following which test positive individuals (hemoglobin concentration $>100 \mathrm{ng}$ per $\mathrm{mL}$ buffer) are offered a screening colonoscopy. One aim of this screening colonoscopy is to detect and remove polyps before their differentiation into malignant tumors. Adenomas

Department of Surgical Gastroenterology, Hospital South West Jutland, Esbjerg, Denmark

Conflict of Interest: None

Correspondence to: Magnus Ploug, Department of Surgical Gastroenterology, Hospital South West Jutland, Finsensgade 35, 6700, Esbjerg, Denmark, e-mail: Magnus.Ploug@rsyd.dk

Received 19 April 2021; accepted 23 June 2021; published online 12 October 2021

DOI: https://doi.org/10.20524/aog.2021.0668 that escape visualization are left in situ and may transition into cancer over time, reducing the effectiveness of the screening program. According to the Danish National screening guidelines, a clean screening colonoscopy (no adenomas or malignant lesions found) will exempt the patient from screening invitations for the following 8 years. This long exemption period leaves any missed adenomas with a substantial time to differentiate. Therefore, it is of utmost importance to identify factors that systematically affect the adenoma detection rate (ADR) and have the potential to improve the quality of screening colonoscopy. The diagnostic quality of the screening colonoscopy, and more specifically its possible variation with the time of day, was the main focus of this study.

In general, the diagnostic quality of the colonoscopy depends on the ability to perform a complete examination, as well as on the ability of the endoscopist to detect lesions [2]. The ADR quantifies the proportion of patients in whom a histologically proven adenoma is found, and the cecal intubation rate (CIR) quantifies the proportion of complete endoscopies. Both ADR and CIR are well-recognized indicators of diagnostic quality and both show an inverse relationship with the risk of interval CRC $[3,4]$. 
CIR and ADR can be affected by procedural factors, including withdrawal time, bowel preparation [4,5], and the experience of the performing endoscopist [6,7]. Additionally, recent studies have found both $\mathrm{ADR}$ and $\mathrm{CIR}$ to be lower in colonoscopies performed in the afternoon compared to those performed before noon [4,8-13]. This "afternoon effect" is seen when endoscopists are working full-day endoscopic shifts, and might be due to mental or physical fatigue on the part of the clinician $[11,12,14]$. In response, scheduling 3-h block shifts or half-day shifts seems to eliminate this afternoon-dependent fall in ADR and CIR [15-17].

Some studies failed to show any time-dependent difference in ADR $[7,18]$, and a recent systematic review [19] synthesizing the results of 5 studies, found no overall timeof-day related difference in ADR. All these studies originated from either the USA or Asia and may not reflect the clinical reality outside these settings. In addition, the reported ADRs of these studies are between $23 \%$ and $35 \%$, considerably lower than the $46 \%$ expected in our screening cohort [20]. Therefore, before implementing workflow changes in response to a potential time-of-day variation in diagnostic quality, our aim was to investigate the variation between morning and afternoon colonoscopies in a European screening setting with endoscopists working traditional full-day endoscopy shifts.

\section{Materials and methods}

This was a single-center registry-based study of colonoscopies performed at our institution between March 2014 and August 2018. Screening records were identified and retrieved from the Danish Colorectal Cancer Screening Database (DCCSD).

Colonoscopy records were included if they contained information on the histopathological examination of removed lesions in order to determine adenoma detection and if they included information on the completeness of the colonoscopy. Records were excluded if the examination had not been performed between 8 a.m. and 4 p.m. Our primary outcome was ADR variation, comparing colonoscopies performed in the morning (8-12 a.m.) with colonoscopies performed in the afternoon (12-4 p.m.).

The time of day the colonoscopy was performed and the level of experience of the endoscopists were obtained locally, while all other data were obtained from the DCCSD registry. Endoscopists were divided into 3 groups based on experience, ranging from group 1 "low experience" (up to $2^{\text {nd }}$ year residency), through group 2 "medium experience" (3rd- $5^{\text {th }}$ year residency, register doctors and trained endoscopy nurses), to group 3 "high experience" (consulting surgeons). In our department, screening colonoscopies are performed by surgical gastroenterologists who typically are assigned to the endoscopy unit between 1 and 4 days a month. The equipment used is Olympus CF-Hq190 colonoscopes.

The outcome variable ADR is a binary variable, depending on the detection of adenomas or not. We used the ADR definition provided by both the European Society of Gastrointestinal Endoscopy and the American Society for
Gastrointestinal Endoscopy, defining ADR as the fraction of patients undergoing screening colonoscopy in whom one or more adenomas are detected [21,22].

The CIR was based on the endoscopy report, thus determined by the endoscopist as either complete or incomplete. In addition to analyzing the CIR, we explored the cause of incompleteness, grouped as either insufficient bowel preparation, patient discomfort, stenosis, other, or no reason specified.

The bowel preparation regimen consisted of MoviPrep ${ }^{\oplus}$ (PEG-3350, Sodium Sulfate, Sodium Chloride, Potassium Chloride, Sodium Ascorbate and Ascorbic Acid for Oral Solution). From 2014 until May 2016 the first dose was given at 4 p.m. and the second at 8 p.m., both on the day prior to the examination. From June 2016, split-dose administration was begun, where the first dose was to be taken at 8 p.m. on the day before the examination and the second dose $4 \mathrm{~h}$ prior to the examination. Because of this change in bowel preparation midway through the study period, analyses of both ADR and CIR were repeated for each period specifically.

\section{Statistical analysis}

Statistical analyses were performed using Stata/IC 16.0 (Stata Corp, Texas, USA). A P-value $<0.05$ was considered statistically significant. For analyses of ADR and CIR, multivariate logistic regression was performed. As explanatory variables in the multivariate regression model, we included all variables likely to influence adenoma detection, and which were available from the DCCSD registry or could be obtained locally. These included: age, sex, FIT value, completeness of the examination (for ADR analysis), and the experience level of the endoscopist. In addition, we performed a univariate analysis of the mean number of polyps and the mean number of adenomas detected, to see if this coincided with the results on ADR.

\section{Results}

Data on 4007 consecutive screening colonoscopies were retrieved from DCCSD. Fig. 1 shows the number of records available for ADR analysis and Table 1 shows the distribution of baseline variables. The distribution of the baseline characteristics was comparable between those who had their screening colonoscopy in the morning and those who had it in the afternoon. Overall ADR was $52.1 \%$. ADR was lower in the morning (50.8\%, $1545 / 3039)$ than in the afternoon $(58.1 \%, 360 / 620)$.

Forty-seven individual endoscopists had performed the procedures, with one endoscopist performing 1246 of them. Cases with missing data on any independent variable were less than $1 \%$, leaving a total of 3648 colonoscopies available for the multivariate regression analysis.

The multivariate regression analysis (Table 2) shows that colonoscopies performed in the afternoon had 1.4 the odds of adenoma detection compared to those performed in the morning (95\% confidence interval 1.17-1.68; $\mathrm{P}<0.001)$. Advanced age, male sex, a greater FIT value, and positive cecal 


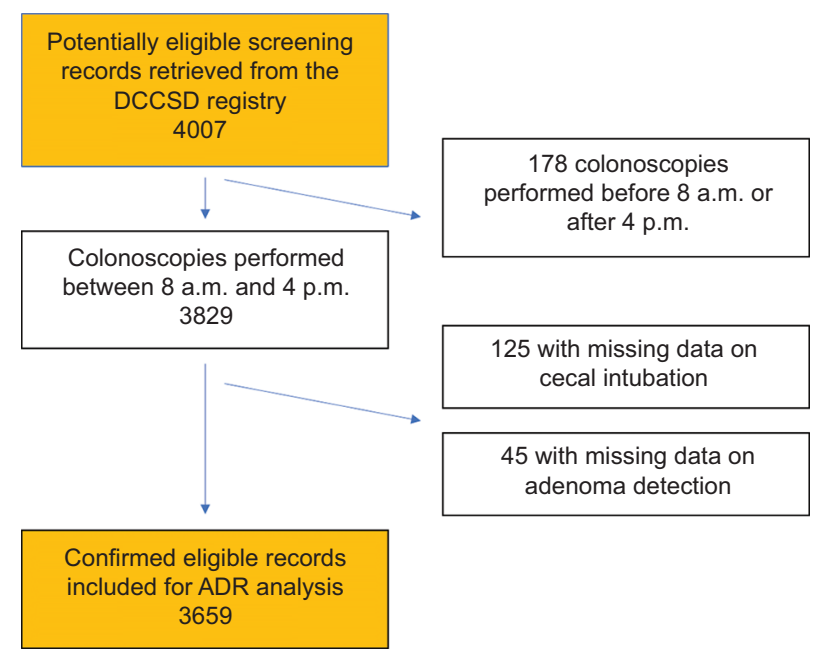

Figure 1: Flow chart showing determination of eligible colonoscopy records DCCSD, Danish Colorectal Cancer Screening Database; $A D R$, adenoma detection rate intubation increased the likelihood of adenoma detection. Repeating the multivariate regression analysis of ADR, using the same explanatory variable but excluding the 1246 procedures performed by one individual endoscopist, yielded similar results. Repeating the multivariate regression analysis on individuals examined either before or after the introduction of split-dose bowel preparation (June 2016) showed identical results, with the afternoon vs. morning odds ratio of ADR being 1.4 before June 2016 and 1.39 after June 2016, both statistically significant.

The mean number of detected polyps was 1.00 in the morning vs. 1.27 in the afternoon, and the mean number of adenomas detected was 0.76 in the morning vs. 0.94 in the afternoon. When ADR was examined on an hourly basis, the lowest ADR was found between 8-9 a.m. (Fig. 2).

The CIR was $95 \%$ (3492/3659) overall, ranging from $95.60 \%$ in the morning (2905/3039), to $94.68 \%$ in the afternoon (587/620). Multivariate logistic regression analysis of CIR (Table 2) showed no significant influence of time of day. Lower age, male sex, lower FIT value, and high endoscopist experience

Table 1 Distribution of baseline characteristics

\begin{tabular}{|c|c|c|c|}
\hline Characteristics & $\begin{array}{l}\text { Morning } \\
\text { (8-12 a.m.) }\end{array}$ & $\begin{array}{l}\text { Afternoon } \\
\text { (12-4 p.m.) }\end{array}$ & $\begin{array}{l}\text { Missing } \\
\text { data, } n\end{array}$ \\
\hline Individuals, $\mathrm{n}(\%)$ & $3039(83)$ & $620(17)$ & - \\
\hline Median age, years (IQR) & $64(57-70)$ & $65(58-70)$ & 0 \\
\hline $\begin{array}{l}\text { Sex } \\
\text { Male, n (\%) } \\
\text { Female, n (\%) }\end{array}$ & $\begin{array}{l}1786(59) \\
1253(41)\end{array}$ & $\begin{array}{l}344(56) \\
276(44)\end{array}$ & 0 \\
\hline Median value FIT test (ng hgb/mL) (IQR) & $284(162-759)$ & $264(163-723)$ & 11 \\
\hline $\begin{array}{l}\text { Endoscopists' experience, n (\% of all endoscopist) } \\
\text { Group 1, "low experience" } \\
\text { Group 2, "medium experience" } \\
\text { Group 3, "high experience" }\end{array}$ & $\begin{array}{c}217(7) \\
735(24) \\
2087(69)\end{array}$ & $\begin{array}{c}62(10) \\
160(26) \\
398(64)\end{array}$ & 0 \\
\hline
\end{tabular}

IQR, interquartile range; FIT, fecal immunochemical test

Table 2 Multivariate logistic regression for adenoma detection rate

\begin{tabular}{|c|c|c|c|c|}
\hline \multirow[t]{2}{*}{ Variables } & \multicolumn{2}{|c|}{$\begin{array}{c}\text { ADR } \\
\text { Observations, } \mathrm{n}=3648\end{array}$} & \multicolumn{2}{|c|}{$\begin{array}{c}\text { CIR } \\
\text { Observations, } \mathrm{n}=3648\end{array}$} \\
\hline & Odds ratio $(95 \% \mathrm{CI})$ & P-value & Odds ratio $(95 \% \mathrm{CI})$ & P-value \\
\hline $\begin{array}{l}\text { Time-of-day } \\
\text { Afternoon }\end{array}$ & $1.40(1.17-1.68)$ & $<0.001$ & $0.88(0.59-1.30)$ & 0.52 \\
\hline Age & $1.02(1.01-1.03)$ & $<0.001$ & $0.98(0.96-0.997)$ & 0.03 \\
\hline Female sex & $0.57(0.50-0.65)$ & $<0.001$ & $0.59(0.43-0.81)$ & 0.001 \\
\hline FIT test & $1.001(1.0003-1.0007)$ & $<0.001$ & $0.9995(0.9991-0.9999)$ & 0.03 \\
\hline $\begin{array}{l}\text { Endoscopists' exp } \\
\text { Medium } \\
\text { High }\end{array}$ & $\begin{array}{c}0.9(0.69-1.20) \\
1.06(0.83-1.37)\end{array}$ & $\begin{array}{l}0.50 \\
0.63\end{array}$ & $\begin{array}{l}1.51(0.90-2.53) \\
2.26(1.40-3.64)\end{array}$ & $\begin{array}{c}0.12 \\
0.001\end{array}$ \\
\hline Cecal intubation & $2.87(2.02-4.07)$ & $<0.001$ & - & - \\
\hline
\end{tabular}

Overall test significance $<0.0001$ (both models)

$A D R$, adenoma detection rate; CIR, cecal intubation rate; CI, confidence interval; FIT, fecal immunochemical test 
all increased the odds of a complete investigation. Repeating the multivariate analysis on colonoscopies performed before or after the introduction of split-dose bowel preparation showed identical results, with CIR not being influenced by time of day.

Regarding the reason for a colonoscopy to be incomplete, this did not differ between morning and afternoon colonoscopies. In particular, no difference was seen in relation to insufficient bowel preparation, which accounted for $1.6 \%(n=48)$ before noon and $2.1 \%(n=13)$ after noon. A total of 4 complications were reported to the registry: one case of bleeding and 3 cases of perforation. This corresponds to a complication rate of $1 \%$ (4/3659). The variation between morning and afternoon was not statistically significant.

\section{Discussion}

In this single-center study of time-of-day variation in diagnostic quality, 3659 consecutive screening colonoscopies were evaluated using multivariate regression analysis. The ADR was significantly higher in afternoon colonoscopies when compared to morning colonoscopies. Advanced age, male sex, a high FIT value and a complete examination all increased the likelihood of adenoma detection, while the experience of the endoscopist had no impact. Time of day had no impact on the CIR.

A greater ADR after midday is in contradiction to our hypothesis, and in contrast to previous studies that showed either a lower $\mathrm{ADR}$ in the afternoon $[8,10-13,15]$ or no time-related differences $[7,16,18]$. Both the mean number of detected polyps and the mean number of detected adenomas were higher in the afternoon, thus substantiating the observed difference in ADR. These results are surprising, and show that similar studies performed in different settings/regions might not have good external validity and that changing clinical practice without generating local/regional data, should be done with care. The lowest ADR was found in the early morning hours, which could warrant future research into whether procedural factors such as poor bowel preparation quality, or possibly even endoscopist-related factors related to the morning hours, could have a causal impact on our observed ADR variation. A subgroup analysis of endoscopies performed

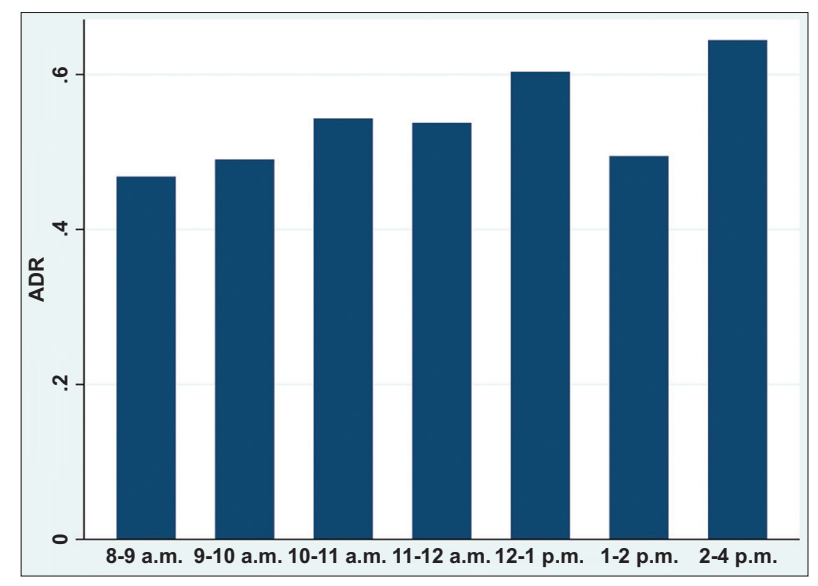

Figure 2 Distribution of the adenoma detection rate (ADR) per hour before or after the introduction of split-dose bowel preparation showed that the difference in ADR between morning and afternoon was equally prevalent in both time periods.

Male sex, advanced age, and higher FIT values were all associated with a greater likelihood of adenoma detection. Males showed roughly double the odds of females for adenoma detection, making sex the exposure variable with the greatest impact on ADR.

Time of day did not statistically impact the CIR, whereas lower age, male sex, and lower FIT value significantly increased the odds of a complete investigation. The most experienced group of endoscopists had double the odds of completing the endoscopy compared to the least experienced group. This implies that nearby support from highly experienced endoscopists should be readily available in every screening setting where younger endoscopists are performing the examinations.

In trying to explain our results on $\mathrm{ADR}$, we considered possible confounders but found none that could plausibly explain our results. Allocation of the patient to either morning or afternoon examination was random, the workload was constant throughout the day and each colonoscopy had equal time reserved for it. The overall lower number of screening colonoscopies in the afternoon does not represent a lower workload but reflects the fact that non-screening colonoscopies have a tendency to be booked in the afternoon. All endoscopists worked full-day endoscopic shifts, and patients received the same regimen for bowel preparation, irrespective of the time the endoscopy was scheduled. The observed equal rate of incomplete colonoscopies morning and afternoon due to insufficient bowel preparation also indicates that bowel preparation quality, in general, was not different between morning and afternoon. This might be a result of the split-dose bowel preparation regimen installed in all screening colonoscopies in 2016.

This was a retrospective single-center study of a large group of consecutive screening colonoscopies. It was based on a national registry with very high data completeness, as is indicated by the exclusion of less than $5 \%$ of potentially eligible cases due to missing information on CIR or ADR. Among the included records, missing information affected less than $1 \%$. However, the study has some limitations. A standardized description of the bowel preparation quality and of the withdrawal time was not available. Information on these variables (as well as data on other potentially modifiable patient- or endoscopist-related factors) would have been of value in exploring why ADR was higher in the afternoon. It would be a natural next step, moving onwards from the results of this study to look at possible modifiable factors such as withdrawal time, endoscopist fatigue, bowel preparation quality, and whether these variables show any timeof-day dependent differences. This study was a single-center study with the inherent limitation of a lack of generalizability due to possible local uniqueness in patient cohort characteristics, endoscopist training, time reserved per endoscopy, workload, and many other factors. Therefore, our results are not intended to be generalized, but instead to highlight the need for local/ regional evaluation and to draw focus towards identifying factors that can systematically affect the quality of a screening program.

In conclusion, the $\mathrm{ADR}$ of screening colonoscopies was highest in the afternoon and patients investigated in the morning 


\section{Summary Box}

\section{What is already known:}

- Adenoma detection rate (ADR) is a well-established quality indicator and is inversely related to the risk of interval cancer

- Studies primarily from the USA and Asia have found ADR to be higher in the morning

- In Denmark, a clean screening colonoscopy exempts the patient from the screening program for the following 8 years

\section{What the new findings are:}

- The overall ADR and cecal intubation rate were high, showing the good quality of the screening examinations as a whole

- ADR was higher in the afternoon than in the morning, even after controlling for age, sex, fecal immunochemical test value, cecal intubation, and the experience of the endoscopist

- Systematic differences in colonoscopy quality should be investigated locally/regionally and any differences found should be evaluated and reduced in order to secure a uniform screening program for all individuals

might thus systematically be put at unnecessary risk of having missed adenomas. Because of the 8-year exemption issued after a clean screening colonoscopy, it is of utmost importance to identify and target such differences. This will have the potential to improve the quality of screening colonoscopies and ensure that each patient receives equal examination. Based on our results, implementing workflow changes to encompass timeof-day variations should be based on local/regional evaluation and care should be taken when extrapolating findings from studies performed in settings different from one's own.

\section{References}

1. Hewitson P, Glasziou P, Irwig L, Towler B, Watson E. Screening for colorectal cancer using the faecal occult blood test, Hemoccult. Cochrane Database Syst Rev 2007;2007:CD001216.

2. Kaminski MF, Thomas-Gibson S, Bugajski M, et al. Performance measures for lower gastrointestinal endoscopy: a European Society of Gastrointestinal Endoscopy (ESGE) quality improvement initiative. United European Gastroenterol J 2017;5:309-334.

3. Wieszczy P, Regula J, Kaminski MF. Adenoma detection rate and risk of colorectal cancer. Best Pract Res Clin Gastroenterol 2017;31:441-446.

4. Wells CD, Heigh RI, Sharma VK, et al. Comparison of morning versus afternoon cecal intubation rates. BMC Gastroenterol 2007;7:19.

5. Wang H, Wang P, Liu X, et al. Factors predicting the colorectal adenoma detection rate in colonoscopic screening of a Chinese population: a prospective study, Medicine (Baltimore) 2019;98:e15103.

6. Chen SC, Rex DK. Endoscopist can be more powerful than age and male gender in predicting adenoma detection at colonoscopy. Am J Gastroenterol 2007;102:856-861.

7. Leffler DA, Kheraj R, Bhansali A, et al. Adenoma detection rates vary minimally with time of day and case rank: a prospective study of 2139 first screening colonoscopies. Gastrointest Endosc 2012;75:554-560.

8. Vicari J. The influence of timing of colonoscopy on adenoma detection: is timing everything? Am J Gastroenterol 2009;104:1666-1667.

9. Sanaka MR, Deepinder F, Thota PN, Lopez R, Burke CA. Adenomas are detected more often in morning than in afternoon colonoscopy. Am J Gastroenterol 2009;104:1659-1664.

10. PaeckKH,Heo WJ, ParkDI, etal. Colonoscopy scheduling influences adenoma and polyp detection rates. Hepatogastroenterology 2013;60:1647-1652.

11. Almadi MA, Sewitch M, Barkun AN, Martel M, Joseph L. Adenoma detection rates decline with increasing procedural hours in an endoscopist's workload. Can J Gastroenterol Hepatol 2015;29:304-308.

12. Chan MY, Cohen H, Spiegel BM. Fewer polyps detected by colonoscopy as the day progresses at a Veteran's Administration teaching hospital. Clin Gastroenterol Hepatol 2009;7:1217-1223.

13. Sanaka MR, Shah N, Mullen KD, Ferguson DR, Thomas C, McCullough AJ. Afternoon colonoscopies have higher failure rates than morning colonoscopies. Am J Gastroenterol 2006; 101:2726-2730.

14. Lee CK, Cha JM, Kim WJ. Endoscopist fatigue may contribute to a decline in the effectiveness of screening colonoscopy. J Clin Gastroenterol 2015;49:e51-e56.

15. Wu J, Zhao SB, Wang SL, et al. Comparison of efficacy of colonoscopy between the morning and afternoon: A systematic review and meta-analysis. Dig Liver Dis 2018;50:661-667.

16. Gurudu SR, Ratuapli SK, Leighton JA, Heigh RI, Crowell MD. Adenoma detection rate is not influenced by the timing of colonoscopy when performed in half-day blocks. Am J Gastroenterol 2011;106:1466-1471.

17. Munson GW, Harewood GC, Francis DL. Time of day variation in polyp detection rate for colonoscopies performed on a 3-hour shift schedule. Gastrointest Endosc 2011;73:467-475.

18. Xu Y, Chen K, Xu L, Yuan X, Wu Y, Chen P. Diagnostic yield is not influenced by the timing of screening endoscopy: morning versus afternoon. Scand J Gastroenterol 2018;53:365-369.

19. Barakat M, Panchal A, Abdelfatah MM, Elhanafi S, Carr-Locke DL, Othman MO. Morning versus afternoon adenoma detection rate: a systematic review and meta-analysis. Eur J Gastroenterol Hepatol 2020;32:467-474.

20. Danish Colorectal Cancer Screening Database (DCCSD). Yearly report 2018. Available from: https://www.rkkp.dk/ siteassets/forside/databaser/arsrapporter/dts-arsrapport2018_ anonymiseret_endelig_marts2020.pdf [Accessed 7 September 2021].

21. Rex DK, Schoenfeld PS, Cohen J, et al. Quality indicators for colonoscopy. Gastrointest Endosc 2015;81:31-53.

22. Kaminski MF, Thomas-Gibson S, Bugajski M, et al. Performance measures for lower gastrointestinal endoscopy: a European Society of Gastrointestinal Endoscopy (ESGE) Quality Improvement Initiative. Endoscopy 2017;49:378-397. 